\title{
Effect of insulin sensitivity on pulsatile insulin secretion
}

\author{
Miloš Žarković, Jasmina Ćirić, Miloš Stojanović, Zorana Penezić, Božo Trbojević, Milka Drezgić \\ and Milica Nešović \\ Institute of Endocrinology, School of Medicine, University of Belgrade, Dr Subotića 13, 11000 Belgrade, Yugoslavia \\ (Correspondence should be addressed to Miloš Žarković; Email: mzarkov@eunet.yu)
}

\begin{abstract}
Objective: The aim of the study was to determine whether derangements in insulin pulsatility are related to the presence of insulin resistance or whether these changes occur only in non-insulindependent diabetes mellitus (NIDDM).

Design and methods: The study included 26 obese, 11 NIDDM and 10 control subjects. The obese group was divided into a low insulin (plasma insulin $<20 \mathrm{mU} / \mathrm{l}$, OLI, 14 subjects) and a high insulin (OHI, 12 subjects) group. For pulsatility analysis blood was sampled every 2 min for $90 \mathrm{~min}$. Pulsatility analysis was carried out using the PulsDetekt program. The insulin secretion randomness was quantified using interpulse interval deviation (IpID) and approximate entropy (ApEn). ApEn and ApEn normalized by S.D. of the individual insulin time series ( $\mathrm{ApEn}$ ) were calculated. Lower values of ApEn and IpID indicate more regular secretion. Homeostasis model assessment (HOMA) was used to quantify insulin sensitivity.

Results: Insulin pulses were significantly less regular in the OHI and the NIDDM groups compared with the control and the OLI groups (control: ApEn 0.54 \pm 0.16 , nApEn 0.69 \pm 0.19 , IpID 2.53 \pm 0.99 ; OLI: ApEn 0.64 \pm 0.12 , nApEn 0.79 \pm 0.15 , IpID 2.92 \pm 1.09 ; OHI: ApEn 0.88 \pm 0.07 , nApEn $0.92 \pm 0.07$, IpID $3.95 \pm 0.84$; NIDDM: ApEn 0.92 \pm 0.16 , nApEn $0.99 \pm 0.09$, IpID $4.41 \pm 0.53$; means \pm s.D.). There was no difference in the pulse regularity between the OHI and the NIDDM groups.

Conclusions: Decrease in insulin sensitivity was correlated with the reduction of insulin secretion regularity. Therefore irregular insulin secretion is related to a reduction in insulin sensitivity, and it is not unique to NIDDM.
\end{abstract}

European Journal of Endocrinology 141 494-501

\section{Introduction}

It is well known that insulin is secreted in a pulsatile manner $(1,2)$. Irregular insulin secretion has been reported in non-insulin-dependent diabetes mellitus (NIDDM) and in 1st degree relatives of both NIDDM and IDDM patients (3-6). A finding common to NIDDM and obesity is reduced insulin sensitivity (7-9). Reduced insulin sensitivity has also been correlated with an increased detected pulse frequency $(10,11)$. Accordingly, impairment of pulsatile insulin secretion would be present in states with reduced insulin sensitivity, regardless of the cause of the reduction in sensitivity. Therefore, secretion disturbances should be present both in NIDDM and insulinresistant obese subjects, but absent in insulin-sensitive obese subjects. The rationale of the present study was to determine whether alterations in the insulin secretory profile (pulsatility) are related to the presence of insulin resistance or whether these changes occur only in states of glucose intolerance and NIDDM.

\section{Materials and methods}

\section{Subjects}

Studies were performed in 26 obese, 11 NIDDM subjects and 10 non-obese healthy subjects (control). Diabetes was diagnosed according to American Diabetes Association criteria (12). The obese group was subdivided into a low fasting plasma insulin group (mean insulin $<20 \mathrm{mU} / \mathrm{l}$ - upper limit of normal range, OLI, 14 subjects) and a high fasting plasma insulin group (mean insulin $>20 \mathrm{mU} / \mathrm{l}$ - upper limit of normal range, $\mathrm{OHI}, 12$ subjects) depending on the mean level of insulin concentration in samples obtained for pulsatility analysis (46 samples). Inclusion criteria for the obese subjects were: (i) body mass index (BMI) greater than $28 \mathrm{~kg} / \mathrm{m}^{2}$, (ii) normal oral glucose tolerance test, (iii) normal renal and liver function tests, (iv) no intercurrent disease in the last three weeks, (v) no signs of endocrine dysfunction, (vi) not taking any drugs, (vii) no history of diabetes mellitus 
Table 1 The metabolic and clinical characteristics of the study groups. Data are means \pm S.D.

\begin{tabular}{|c|c|c|c|c|c|}
\hline & \multicolumn{4}{|c|}{ Non NIDDM } & \multirow{2}{*}{$\begin{array}{c}\text { NIDDM } \\
\text { All }\end{array}$} \\
\hline & All & Control & $\begin{array}{c}\text { Obese } \\
\text { low insulin }\end{array}$ & $\begin{array}{c}\text { Obese } \\
\text { high insulin }\end{array}$ & \\
\hline Age (years) & $36.25 \pm 9.60$ & $34.90 \pm 11.20 \dagger$ & $37.29 \pm 11.02 \dagger$ & $36.17 \pm 6.66 \dagger$ & $50.91 \pm 9.63^{*}$ \\
\hline Body mass index $\left(\mathrm{kg} / \mathrm{m}^{2}\right)$ & $32.09 \pm 8.96$ & $20.95 \pm 1.81 \dagger$ & $34.06 \pm 3.47^{\star}$ & $39.09 \pm 8.22^{*}$ & $35.98 \pm 6.91^{*}$ \\
\hline Plasma insulin (mU/l) & $19.22 \pm 13.91$ & $10.99 \pm 5.00 \dagger$ & $10.91 \pm 4.87 \dagger$ & $35.76 \pm 10.93^{*}$ & $33.82 \pm 21.58^{*}$ \\
\hline Plasma glucose (mmol/l) & $4.82 \pm 0.55$ & $4.42 \pm 0.34 \dagger$ & $4.75 \pm 0.51 \dagger$ & $5.23 \pm 0.47 \dagger$ & $10.68 \pm 5.27^{*}$ \\
\hline HOMA sensitivity (\%) & $37.05 \pm 25.67$ & $52.95 \pm 29.42 \dagger$ & $46.26 \pm 18.57 \dagger$ & $13.05 \pm 3.76^{*}$ & $11.86 \pm 7.87^{*}$ \\
\hline HOMA $\beta$-cell function (\%) & $417.90 \pm 257.48$ & $425.43 \pm 260.59 \dagger$ & $280.18 \pm 174.19 \dagger$ & $572.29 \pm 263.32 \dagger$ & $124.32 \pm 83.61^{*}$ \\
\hline
\end{tabular}

${ }^{*} P<0.05$ compared with control group, $\uparrow P<0.05$ compared with NIDDM group.

in 1st degree relatives. Inclusion criteria for NIDDM subjects were: (i) negative urinary ketones, (ii) normal renal and liver function tests, (iii) no intercurrent disease in the last three weeks, (iv) no signs of other endocrine dysfunction, (v) not taking any drugs. All NIDDM subjects were treated with diet alone or were newly diagnosed. Inclusion criteria for the control group were the same as for the obese group except that BMI was greater than 19 and less than $24 \mathrm{~kg} / \mathrm{m}^{2}$ and that the mean level of insulin concentration in samples obtained for pulsatility analysis was less than $20 \mathrm{mU} / \mathrm{l}$. The metabolic and clinical characteristics of the study groups are detailed in Table 1. Representative insulin profiles are presented in Fig. 1. A local ethical committee approved all studies. All subjects gave informed consent.

\section{Study protocol}

Subjects were admitted to the Institute of Endocrinology, Belgrade School of Medicine. They consumed standardized isocaloric meals (25-30 kcal/ $/ \mathrm{kg}$ ), composed of $55 \%$ carbohydrate, $30 \%$ fat and $15 \%$ protein over the $48 \mathrm{~h}$ before the study. After a 12-h fast, blood was sampled for insulin determination. An indwelling venous catheter was placed in the antecubital vein. Sampling started at $08.00 \mathrm{~h}$, and lasted for $90 \mathrm{~min}$ with an intersample interval of $2 \mathrm{~min}$. Blood was collected using a plastic syringe, and transferred to plain glass tubes. Heparin was not used. Before taking a blood sample, $0.2 \mathrm{ml}$ (catheter volume) was taken with another syringe and discarded. The glucose samples were measured immediately after sampling, while insulin samples were frozen at $-20{ }^{\circ} \mathrm{C}$ until assayed (maximum time frozen one week).

\section{Pulse analysis}

Pulse analysis was carried out using the PulsDetekt program that is based on the deconvolution of secretory and metabolic events. Details of the PulsDetekt program algorithm are given in the Appendix.

\section{PulsDetekt program validation}

Simulated series To validate the PulsDetekt program 135 synthetic time series were generated by computer simulation with the following characteristics: (i) increase in hormone concentration followed by monoexponential decay, (ii) sampling length 90 time units, (iii) intersample interval 0.1 time unit, (iv) halflife of monoexponential decay 8 time units, (v) pulse amplitude of 10,20 , and 40 units with a coefficient of variation of $20 \%$, (vi) interpulse interval of 6,10 and 15 time units with a coefficient of variation of $20 \%$, (vii) normally distributed noise, 5, 10, and 20\% of series value at a given point in time. Each series was resampled to intersample interval of 1,2 and 3 time units, thus generating 3 new series. The PulsDetekt program correctly predicted synthetic series parameters (half-life, number of pulses, interpulse interval and pulse amplitude). The program also proved to be robust to noise increase, but a reduction in the sampling rate from two to three units led to a significant increase in the false negative rate (false positive rate: $0.00 \%$ at noise level $5 \%$ and sample interval 1 unit to $0.49 \%$ at noise level $20 \%$ and sample interval 3 units; false negative rate: $0.00 \%$ at noise level $5 \%$ and sample interval 1 unit to $13.58 \%$ at noise level $20 \%$ and sample interval 3 units).

Validation in vivo Our method of pulse detection was validated by the assessment of insulin concentration profiles during suppression of the endogenous insulin secretion. To simulate pulsatile secretion insulin was given in i.v. boluses. Four healthy subjects were included in the study. Suppression of endogenous insulin secretion was achieved using octreotide, $0.5 \mu \mathrm{g} / \mathrm{min}$, preceded by $25 \mu \mathrm{g}$ bolus (13). Blood was sampled for $90 \mathrm{~min}$ with an intersample interval of $2 \mathrm{~min}$. The octreotide bolus was given at time 0 , and the continuous infusion was given during the whole sampling period. At 30, 40, 50, 60, 70 and $80 \mathrm{~min} 72 \mathrm{mU}$ insulin (Actrapid HM, Novo Nordisk, Bagsvaerd, Denmark) boluses were given into a cubital vein, contralateral to the sampling one. The insulin bolus was calculated to give a pulse amplitude of about $10 \mathrm{mU} / \mathrm{l}$. Detected 


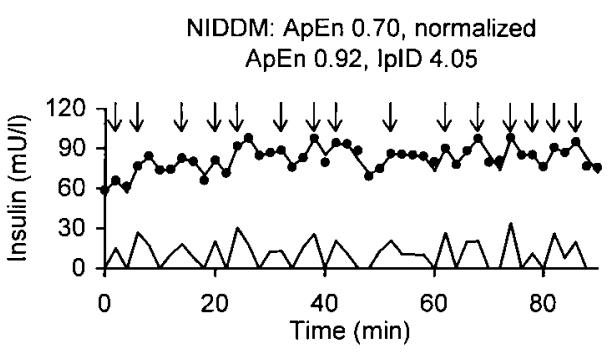

OHI: ApEn 0.96,

normalized ApEn 0.97, IpID 4.24

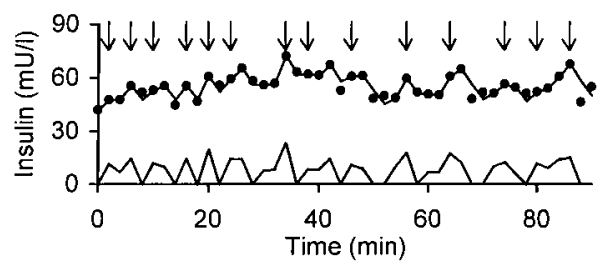

OLI: ApEn 0.63,

normalized ApEn 0.70, IpID 1.86

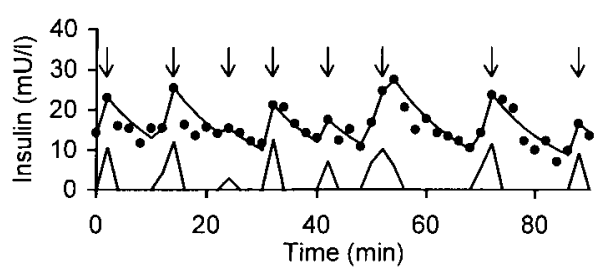

Control: ApEn 0.35,

normalized ApEn 0.49, IpID 0.75

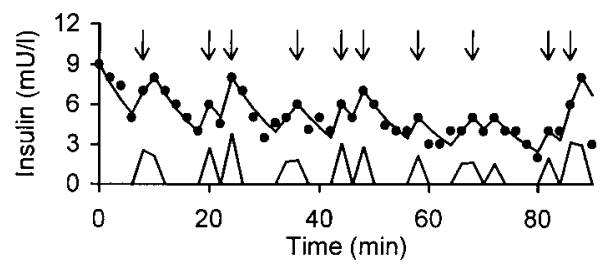

NIDDM: ApEn 0.95, normalized ApEn 1.02, IpID 4.98

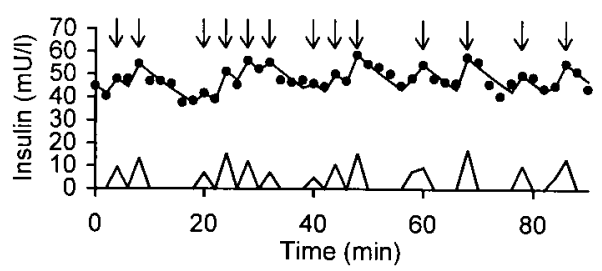

OHI: ApEn 0.98,

normalized ApEn 0.92, IpID 4.85

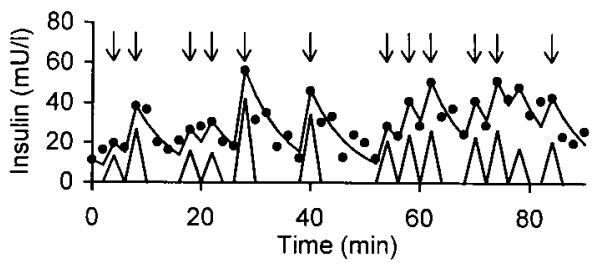

OLI: ApEn 0.71 ,

normalized ApEn 0.77, IpID 2.05

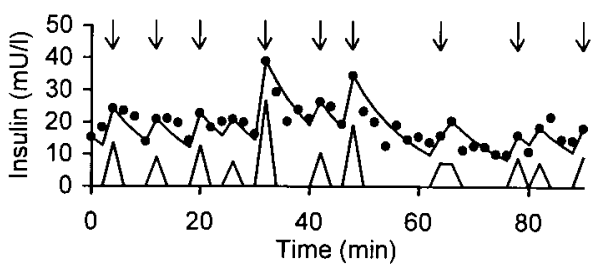

Control: ApEn 0.50,

normalized ApEn 0.56, IpID 2.80

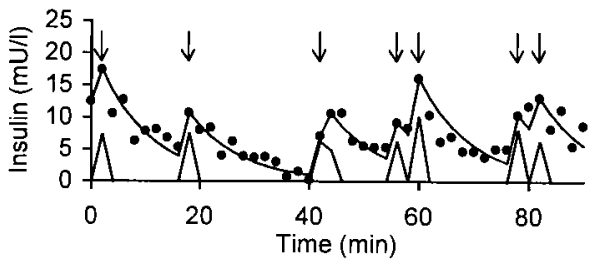

Figure 1 Representative insulin profiles for each group. Each significant pulse is identified by an arrow. Measured data are represented by dots, and lines represent calculated data (fit and deconvolution).

insulin pulses were assessed for temporal correlation with the pulses of exogenous insulin. From 30 min of sampling only exogenous pulses were detected. All but one pulse of exogenous insulin were detected (false negative: 1 (4.2\%); false positive 0$)$. On visual inspection (subject 2) at the time point where the undetected pulse was expected only a small increase in the insulin concentration was found $(1.4 \mathrm{mU} / \mathrm{l})$ which was not significant enough to be detected as a pulse (Fig. 2).

\section{Assays}

Glucose was determined using a Beckman glucose analyzer. The precision of the glucose determination was $0.17 \mathrm{mmol} / \mathrm{l}$. Insulin was determined using a radioimmunoassay (INEP, Zemun, Yugoslavia). All samples from one subject were processed in the same batch. The intra-assay coefficient of variation was $5.8 \%$. The minimal detectable concentration of insulin was $1 \mathrm{mU} / \mathrm{l}$.

\section{Data analysis}

Approximate entropy Approximate entropy (ApEn) analysis was used to quantify the degree of randomness of insulin secretion (14). ApEn determines the likelihood that a certain secretory pattern will be the same throughout the sampling period, although the pulses contained in that pattern can be irregularly spaced. ApEn is a relative measure of system randomness, and a higher approximate entropy value represents higher system randomness. Approximate entropy was computed according to the program described by Pincus 

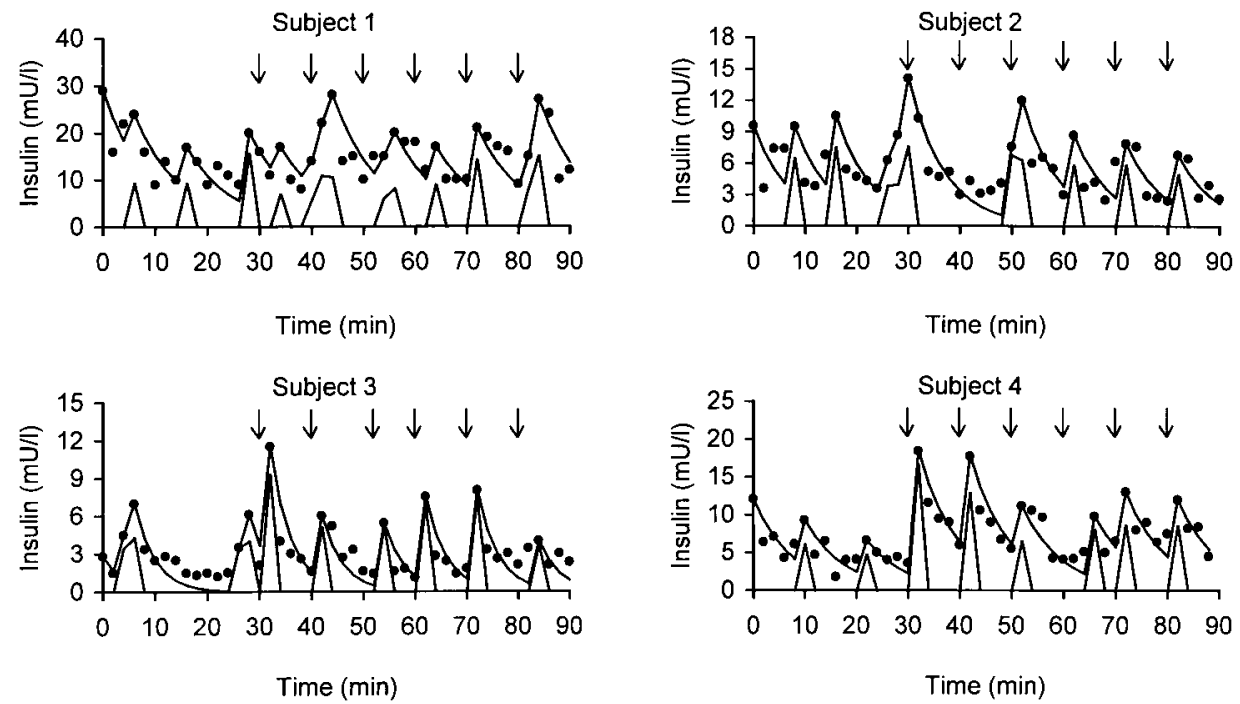

Figure 2 Validation data. All four subjects are presented. Arrows represent times when insulin boluses were administered. Measured data are represented by dots, and lines represent calculated data (fit and deconvolution).

et al. (15). We calculated two sets of ApEn values: (i) $\mathrm{m}=1$ and $r=1.5 \mathrm{mU} / \mathrm{l}$ and (ii) $\mathrm{m}=1$ and $r=20 \%$ of S.D. of the individual subject insulin time series. In choosing the $r$ input parameter in ApEn as a fixed percentage of each data set's S.D. we normalize each epoch by its overall S.D. By calculating ApEn and normalized ApEn we quantify amplitude (ApEn) vs frequency (normalized ApEn) time series irregularities from a separate point of view (16).

Interpulse interval deviation Interpulse interval deviation (IpID) is the deviation from expected interpulse interval (EIpI) if all the pulses were perfectly regularly spaced. It was calculated according to the following equation where $\mathrm{n}$ is the total number of samples and i is a sample indexing term:

$$
\mathrm{IpID}=\frac{\sum_{j=1}^{n}\left|\operatorname{IpI}_{i}-\mathrm{EIpI}\right|}{\text { EIpI }}
$$

The EIpI was calculated as the time between the first and last detected pulse divided by the number of interpulse intervals.

Insulin sensitivity quantification To quantify insulin sensitivity, homeostasis model assessment (HOMA) was used. HOMA is a mathematical model of insulin/glucose interactions that estimates a set of insulin sensitivity and $\beta$-cell function that is expected to give the fasting glucose and insulin concentrations observed in the subject. Results are expressed as a percentage of the values found in a reference population (young, fit subjects with an ideal body weight) $(17,18)$.
Statistical analysis Statistical analyses were carried out on all four groups (control, OLI, OHI, NIDDM), in aggregated groups (non-diabetic vs diabetic), and in all subjects, to assess the influence of insulin sensitivity and diabetic state of the subject.

Groups were compared using ANOVA. For variables with unequal variances the Welch procedure was used. For multiple comparisons the Games-Howell and Dunnet test were used. The Pearson correlation was used to assess correlation between variables. Multivariate regression and partial correlation were used to assess influence of multiple variables (19). The results are expressed as means \pm standard deviation (s.D.). $P$ values less than 0.05 were considered to be statistically significant.

\section{Results}

Insulin sensitivity was similar in the OLI and control groups. Insulin sensitivity was also similar in the $\mathrm{OHI}$ and the NIDDM groups. However, insulin sensitivity was significantly lower in the NIDDM and OHI groups than in the OLI and control groups. The $\beta$-cell function measured by HOMA was impaired in the NIDDM compared with the other three groups (Table 1).

The insulin half-life in peripheral circulation determined by the PulsDetekt program did not differ between the groups (control 10.20 \pm 3.56 ; OLI 9.03 \pm 4.61 ; OHI $8.79 \pm 4.79$; NIDDM $8.01 \pm 4.70$ min). However, insulin pulse amplitude was significantly smaller in the control and the OLI group compared with the other two groups (control 5.37 \pm 1.97 , OLI $5.56 \pm$ 2.92, OHI $13.26 \pm 5.08$, NIDDM $13.38 \pm 7.11 \mathrm{mU} / \mathrm{l})$. There was no difference in insulin pulse amplitude between the $\mathrm{OHI}$ and the NIDDM groups. The number 
Table 2 Characteristics of pulsatile insulin secretion. Data are means \pm S.D.

\begin{tabular}{lcccr}
\hline & Control & OLI & OHI & NIDDM \\
\hline Insulin half-life (min) & $10.20 \pm 3.56$ & $9.03 \pm 4.61$ & $8.79 \pm 4.79$ & $8.01 \pm 4.70$ \\
Number of pulses per 90 min & $9.60 \pm 1.84 \dagger$ & $11.21 \pm 3.02 \dagger$ & $12.75 \pm 1.48^{*}$ & $13.82 \pm 1.47^{*}$ \\
Amplitude (mU/l) & $5.37 \pm 1.97 \dagger$ & $5.56 \pm 2.92 \dagger$ & $13.26 \pm 5.08^{*}$ & $13.38 \pm 7.11^{*}$ \\
Amplitude as a \% of mean level & $53.02 \pm 18.09$ & $52.38 \pm 18.53$ & $39.15 \pm 17.70$ & $43.48 \pm 14.82$ \\
Interpulse interval (min) & $9.72 \pm 2.08 \dagger$ & $8.72 \pm 3.02$ & $7.08 \pm 0.87^{*}$ & $6.53 \pm 0.62^{*}$ \\
Approximate entropy & $0.54 \pm 0.16 \dagger$ & $0.64 \pm 0.12 \dagger$ & $0.88 \pm 0.07^{*}$ & $0.92 \pm 0.16^{*}$ \\
Normalized approximate entropy & $0.69 \pm 0.19 \dagger$ & $0.79 \pm 0.15 \dagger$ & $0.92 \pm 0.07^{*}$ & $0.99 \pm 0.09^{*}$ \\
Interpulse interval deviation & $2.53 \pm 0.99 \dagger$ & $2.92 \pm 1.09 \dagger$ & $3.95 \pm 0.84^{*}$ & $4.41 \pm 0.53^{*}$ \\
\end{tabular}

${ }^{*} P<0.05$ compared with control group, $\dagger P<0.05$ compared with NIDDM group.

of detected pulses was increased and therefore the calculated interpulse interval was significantly shorter in the NIDDM and the OHI groups compared with the control group (number of pulses per 90 min: control $9.60 \pm 1.84$, OLI $11.21 \pm 3.02$, OHI $12.75 \pm 1.48$, NIDDM 13.82 \pm 1.47 ; interpulse interval: control $9.72 \pm 2.08$, OLI $8.72 \pm 3.02$, OHI $7.08 \pm 0.87$, NIDDM $6.53 \pm 0.62 \mathrm{~min})$. In respect to these parameters the OLI and the control group did not differ (Table 2).

In the analysis of all subjects, as well as in that of aggregated groups insulin pulse amplitude and the number of insulin pulses were correlated with insulin sensitivity (pulse amplitude: all subjects $r=-0.678$, $P<0.001$, non diabetic group $r=-0.694, P<0.001$, NIDDM group $r=-0.700, P=0.017$; number of insulin pulses: all subjects $r=-0.473, P=0.001$, non-diabetic group $r=-0.354, P=0.039$, NIDDM group $r=-0.642, P=0.033$ ).

ApEn and IpID were used as measures of randomness of insulin secretion. Lower values of ApEn and IpID indicate more regular secretion. All measures of insulin pulse regularity (ApEn, normalized ApEn and IpID) were significantly higher in the OHI and the NIDDM groups compared with the control group. The control group and the OLI group did not differ. There was no significant difference in measures of insulin pulse regularity between the $\mathrm{OHI}$ and the NIDDM groups (Table 2).
To evaluate the possible impact of age on regularity of insulin pulses multiple regression of measures of randomness vs age, mean glucose level and mean insulin level was performed. All subjects were included in the analysis. Age and mean glucose level did not significantly influence any of the measures of insulin pulse regularity.

ApEn and normalized ApEn were significantly negatively correlated with insulin sensitivity, both in all subject analyses and in the aggregated groups analysis. However, IpID was significantly correlated with insulin sensitivity in all subjects analysis and in the non-diabetic group analysis, but not in the diabetic group analysis. Controlling correlation for blood glucose did not change the significance of any of the studied relations (Table 3 and Fig. 3).

\section{Discussion}

In the present study, we sought to determine whether alterations in the pulsatile secretion of insulin are related to the presence of insulin resistance or whether these changes occur only in states of glucose intolerance and NIDDM. We chose to compare insulin sensitive (control and OLI) vs insulin resistant (OHI and NIDDM) subjects in order to demonstrate the effect of insulin resistance per se on the regularity of high frequency oscillation of the insulin secretion. A deconvolution

Table 3 Correlation coefficient (first row) and partial correlation coefficient (controlled for glucose) (second row) with insulin sensitivity.

\begin{tabular}{lccc}
\hline & \multicolumn{2}{c}{ Insulin sensitivity } \\
\cline { 2 - 3 } & All subjects & Non-diabetic subjects & NIDDM subjects \\
\hline Approximate entropy & $-0.845^{*}$ & $-0.848^{*}$ & $-0.756^{*}$ \\
Normalized approximate entropy & $-0.819^{*}$ & $-0.821^{*}$ & $-0.825^{\star}$ \\
Interpulse interval deviation & $-0.744^{*}$ & $-0.687^{*}$ & $-0.656 \dagger$ \\
& $-0.701^{*}$ & $-0.648^{*}$ & $-0.688 \dagger$ \\
& $-0.654^{*}$ & $-0.654^{*}$ & 0.250 \\
\end{tabular}

$P$ represents significance of the (partial) correlation coefficient. ${ }^{*} P<0.01,+P<0.05$. 

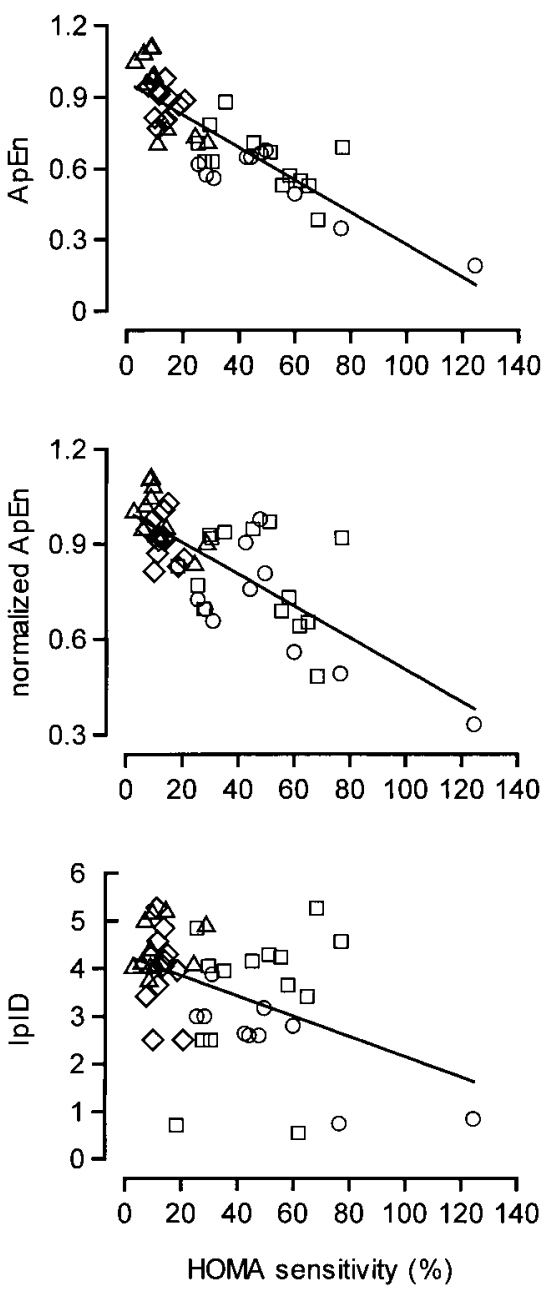

Figure 3 Relation between the approximate entropy (ApEn), the normalized approximate entropy (nApEn), the interpulse interval deviation (IpID), and the HOMA insulin sensitivity. O, control; $\diamond, \mathrm{OHI} \square, \mathrm{OLI} ; \triangle$, NIDDM.

based on the pulse detection program PulsDetekt was used to obtain a series of noise-free insulin pulses. Secretion randomness of those series was determined using two different measures of secretion irregularity (ApEn and IpID).

The insulin half-life determined in this study was comparable to previously published data, where it was determined by a range of different methods (20-22). The frequency of insulin pulses found in the present study was higher and the interpulse interval was shorter (non-diabetic group $11.3 \pm 2.5$ pulses $/ 90 \mathrm{~min}$; $8.4 \pm 2.4 \mathrm{~min}$ ) than some older studies (interpulse interval 11-13 min) $(1,3,4)$, and similar to more recent studies (number of insulin pulses $11.8 \pm 0.9$ per $90 \mathrm{~min}$; interpulse interval 4.1 to $6.5 \mathrm{~min})(10,23)$. Probably the principal cause of this difference is the pulse detection methodology. Earlier studies used autocorrelation or spectral analysis, while more recent studies, including ours, used heuristic methods of pulse detection. Autocorrelation and spectral analysis are not suited for hormone series analysis due to the assumption of absolutely regular pulses and lack of resolution and precision for short time series. Spectral density function of a noise-free series sampled at $1 \mathrm{~min}$ for $150 \mathrm{~min}$ would have a resolution of about $5 \mathrm{~min}$ at 95\% confidence level (24). Heuristic methods make no assumptions on pulse regularity and are not dependent on sampling length.

In the previous studies a strong correlation ( $r$ about -0.7) was found between insulin sensitivity and the number of insulin pulses in healthy and NIDDM persons $(10,11)$. We found a smaller but significant correlation between the insulin sensitivity and the number of insulin pulses (all subjects $r=-0.473, P=$ 0.001; non-diabetic $r=-0.354, P=0.039$; NIDDM $r=-0.642, P=0.033)$. The difference between our and previous studies could be due to different methods of pulse detection or to the different methods of insulin sensitivity determination. In previous studies insulin sensitivity was determined using the glucose clamp technique. Results from the insulin clamp are expressed in absolute units, while HOMA results are expressed in relative units.

We found a significant difference in the insulin pulse regularity between the groups with low insulin (control, OLI) and the groups with high insulin (OHI, NIDDM). There was no difference in the insulin pulse regularity between the $\mathrm{OHI}$ and the NIDDM groups. Also, measures of the insulin pulse regularity were correlated with the insulin sensitivity, even after controlling for glucose concentration. The reduced insulin sensitivity causes the increased blood insulin. A higher insulin level means a higher insulin pulse amplitude, and due to the properties inherent to the pulse detection method, it may result in more pulses detected. However, more pulses detected do not necessarily equal more pulse irregularity. The pulse irregularity can be defined as a measure of inequality of interpulse intervals, and/or pulse amplitudes. On the other hand, ApEn determines the likelihood that a certain secretory pattern will be the same throughout the sampling period. If that secretory pattern repeats more often, the number of pulses will be increased, but they will be regular, so ApEn will not increase. On the other hand, if we have a small number of distinctly different secretory patterns, we will have a small number of pulses, but they will be irregular. Thus, increased number of detected pulses does not equal more irregular pulses. Therefore, loss of regular insulin pulsatility is not unique to NIDDM, but is associated with the insulin resistant state or the over-worked $\beta$-cell.

Glucose, somatostatin, and glucagon modify insulin pulse amplitude but not frequency $(23,25,26)$. Therefore, the changes in pulse frequency might be caused by changes in insulin concentration. Pancreatic islets are widely dispersed within the exocrine pancreas. 
Coordinate pulsatile insulin secretion by these dispersed islets is initiated by an intrapancreatic pacemaker and propagated through the intrapancreatic neural network (27). In the transplanted intrahepatic islets, islet reinervation is necessary for the establishment of coordinate pulsatile insulin secretion (28). Therefore, an intrinsic pancreatic neural network is responsible for the coordination of islet secretion $(29,30)$. Inhibition or stimulation of insulin secretion is mediated by the autonomic nervous system (31-34). Insulin influences autonomous nervous system activity, predominantly increasing sympathetic and decreasing parasympathetic activity (35-37). Thus, less regular insulin secretion found in insulin-resistant subjects could be the consequence of changes in intrapancreatic neural network activity.

In conclusion, the present study indicates that a loss of periodic insulin secretion is related to a reduction in insulin sensitivity, and that irregular insulin secretion is not unique to non-insulin-dependent diabetes. Because of the relation of insulin secretion and insulin resistance, elucidation of the mechanisms regulating them may be achieved by studies focusing on pulsatile pancreatic hormone secretion.

\section{Appendix}

\section{PulsDetekt program alogorithm}

The PulsDetekt program is based on the deconvolution of secretory and metabolic events. Metabolic events are considered to follow a monoexponential curve. Standard deviation (S.D.) of the hormone concentration was assumed to be the same as the assay S.D. that was calculated as a polynomial function $(0,1$ or 2 order) of the measured value $(38,39)$.

Hormone concentration at any time $\left(\mathrm{H}_{\mathrm{ti}}\right)$ can be represented as:

$$
H_{t_{i}}=H_{t_{i-1}} e^{k \Delta t}+S_{t_{i}} \delta+\varepsilon_{t_{i}}
$$

where $\mathrm{H}_{\mathrm{t}_{\mathrm{i}-1}}$ is the hormone concentration at previous time, $\mathrm{e}$ is the natural base, $k$ is the elimination rate constant, $\Delta \mathrm{t}$ is the time interval between $\mathrm{t}_{\mathrm{i}}$ and $\mathrm{t}_{\mathrm{i}-1}, \mathrm{~S}_{\mathrm{t}_{\mathrm{i}}}$ is the quantity of hormone secreted from time $t_{i-1}$ to $t_{i}, \delta$ is the Kronecker delta (unit pulse function) and $\varepsilon_{\mathrm{t}_{\mathrm{i}}}$ is the error term that is assumed to be normally distributed. For simplicity we will omit $\delta$ and $\varepsilon_{\mathrm{t}_{\mathrm{i}}}$ from the following equations, and consider $S_{t_{i}}$ as the quantity of hormone secreted at time $t_{i}$, expressed in the same units as $\mathrm{H}_{\mathrm{t}_{\mathrm{i}}}$. Equal sampling intervals are not assumed. The iterative algorithm determines the quantity of hormone secreted $\left(S_{t_{i}}\right)$ and the elimination rate constant (k) minimizing difference between measured and predicted hormone concentrations:

$$
\hat{S}_{t_{i}}=H_{t_{i}}-H_{t_{i-1}} e^{k \Delta t}
$$

and

$$
\hat{\mathrm{H}}_{\mathrm{t}_{\mathrm{i}}}=\hat{\mathrm{S}}_{\mathrm{t}_{\mathrm{i}}}+\mathrm{H}_{\mathrm{t}_{\mathrm{i}-1} \mathrm{e}^{\mathrm{k} \Delta \mathrm{t}}}
$$

Predicted values are marked with the 'hat'. Pulse detection is performed after the optimal value of elimination rate constant $(k)$ is determined. Measured values of the hormone $\left(\mathrm{H}_{\mathrm{t}_{\mathrm{i}}}\right)$ and expected values at time $t_{i}$ if no secretion occurred $\left(\mathrm{H}_{\mathrm{t}_{\mathrm{i}-1}} \mathrm{e}^{\mathrm{k} \Delta \mathrm{t}}\right)$ are compared. If the measured value of the hormone is significantly greater than expected a secretion with amplitude of $S_{t_{i}}$ is considered to have occurred. A significant increase in $S_{t_{i}}$ compared with previous values of $S_{t}$ is considered to represent pulse. Since pulse width would depend on the sampling interval, the program reports only pulse amplitude, but not pulse width.

\section{References}

1 Lang DA, Matthews DR, Peto J \& Turner RC. Cyclic oscillations of basal plasma glucose and insulin concentrations in human beings. New England Journal of Medicine 1979301 1023-1027.

2 Stagner JI, Samols E \& Weir GC. Sustained oscillations of insulin, glucagon, and somatostatin from the isolated canine pancreas during exposure to a constant glucose concentration. Journal of Clinical Investigation 198065 939-942.

3 Lang DA, Matthews DR, Burnet M \& Turner RC. Brief, irregular oscillations of basal plasma insulin and glucose concentrations in diabetic man. Diabetes $198130435-439$.

4 O'Rahilly S, Turner RC \& Matthews DR. Impaired pulsatile secretion of insulin in relatives of patients with non-insulindependent diabetes. New England Journal of Medicine 1988348 1225-1230.

5 Schmitz O, Porksen N, Nyholm B, Skjaerbaek C, Butler PC, Veldhuis JD et al. Disorderly and nonstationary insulin secretion in relatives of patients with NIDDM. American Journal of Physiology 1997272 E218-E226.

6 Bingley PJ, Matthews DR, Williams AJ, Bottazzo GF \& Gale EA. Loss of regular oscillatory insulin secretion in islet cell antibody positive non-diabetic subjects. Diabetologia $19923532-38$

7 DeFronzo RA, Bonadonna RC \& Ferrannini E. Pathogenesis of NIDDM. A balanced overview. Diabetes Care $199215318-$ 368 .

8 Dinneen S, Gerich J \& Rizza R. Carbohydrate metabolism in non-insulin-dependent diabetes mellitus. New England Journal of Medicine 1992327 707-713.

9 Moller DE \& Flier JS. Insulin resistance - mechanisms, syndromes, and implications. New England Journal of Medicine 1991325 938-948.

10 Hunter SJ, Atkinson AB, Ennis CN, Sheridan B \& Bell PM. Association between insulin secretory pulse frequency and peripheral insulin action in NIDDM and normal subjects. Diabetes $199645683-686$.

11 Peiris AN, Stagner JI, Vogel RL, Nakagawa A \& Samols E. Body fat distribution and peripheral insulin sensitivity in healthy men: role of insulin pulsatility. Journal of Clinical Endocrinology and Metabolism 199275 290-294.

12 American Diabetes Association. Clinical practice recommendations 1996. Diabetes Care 199619 (Suppl 1) S1-118.

13 Pei D, Jones CNO, Bhargava R, Chen YDI \& Reaven GM. Evaluation of octreotide to assess insulin-mediated glucose disposal by the insulin suppression test. Diabetologia 199437 843-844.

14 Pincus SM \& Keefee DL. Quantification of hormone pulsatility via an approximate entropy algorithm. American Journal of Physiology 1992262 E741-E754. 
15 Pincus SM, Gladstone IM \& Ehrenkranz RA. A regularity statistics for medical data analysis. Journal of Clinical Monitoring 19917 335-345.

16 Siragy HM, Vieweg WVR, Pincus S \& Veldhuis JD. Increased disorderliness and amplified basal and pulsatile aldosterone secretion in patients with primary aldosteronism. Journal of Clinical Endocrinology and Metabolism 199580 28-33.

17 Matthews DR, Hosker JP, Rudenski AS, Naylor BA, Treacher DF \& Turner RC. Homeostasis model assessment: insulin resistance and beta-cell function from fasting plasma glucose and insulin concentration in man. Diabetologia 198528 412-419.

18 Turner RC, Rudenski AS, Holman RR, Matthews DR \& O'Rahilly SP. Quantitative modelling of endocrine diseases as exemplified by diabetes. Clinical Endocrinology 198726 107-116.

19 Howell DC. Statistical Methods for Psychology, edn 4. Belmont: Duxbury Press, 1997.

20 Dreyer M, Matthaei S, Kuhnau J \& Rudiger HW. Prolonged plasma half-life of insulin in patients with a genetic defect of high affinity binding sites. Hormone and Metabolic Research 1986 $18247-249$.

21 Lanng S, Thorsteinsson B, Roder ME, Nerup J \& Koch C. Insulin sensitivity and insulin clearance in cystic fibrosis patients with normal and diabetic glucose tolerance. Clinical Endocrinology $199441217-223$.

22 Sodoyez JC, Sodoyez Goffaux F, Guillaume M \& Merchie G. ${ }^{123}$ I]Insulin metabolism in normal rats and humans: external detection by a scintillation camera. Science 1983219 865-867.

23 Storch MJ, Rossle M \& Kerp L. Pulsatile Insulinsekretion in die Pfortader bei Leberzirrhose. Deutsche Mediziniche Wochenschrift $1993118134-138$

24 Priestley MB. Spectral analysis in practice. In Spectral Analysis and Time Series, edn 1, ch 7, pp 502-612. Ed MB Priestley. London: Academic Press, 1987.

25 Marchetti P, Scharp DW, Mclear M, Gingerich R, Finke E, Olack B et al. Pulsatile insulin secretion from isolated human pancreatic islets. Diabetes $199443827-830$.

26 Porksen N, Munn SR, Steers JL, Veldhuis JD \& Butler PC. Effects of somatostatin on pulsatile insulin secretion: elective inhibition of insulin burst mass. American Journal of Physiology 1996270 E1043-E1049.

27 Stagner JI \& Samols E. Role of intrapancreatic ganglia in regulation of periodic insular secretions. American Journal of Physiology 1985248 E522-E530.
28 Porksen N, Munn S, Ferguson D, O'Brien T, Veldhuis JD \& Butler P. Coordinate pulsatile insulin secretion by chronic intraportally transplanted islets in the isolated perfused rat liver. Journal of Clinical Investigation 199494 219-227.

29 Opara EC \& Go VL. Effect of nerve blockade on pulsatile insulin and glucagon secretion in vitro. Pancreas 19916 653-658.

30 Stagner JI \& Samols E. Perturbation of insulin oscillations by nerve blockade in the in vitro canine pancreas. American Journal of Physiology 1985248 E516-E521.

31 Atkinson RL, Dahms WT, Bray GA \& Sperling MA. Adrenergic modulation of glucagon and insulin secretion in obese and lean humans. Hormone and Metabolic Research 198113 249-253.

32 Ejiri K, Taniguchi H, Ishihara K, Hara Y \& Baba S. Possible involvement of cholinergic nicotinic receptor in insulin release from isolated rat islets. Diabetes Research and Clinical Practice 1990 8 193-199.

33 Guillot E, Coste A \& Angel I. Alpha 2-adrenoceptors and the regulation of glucose, insulin and amylin levels in diabetic rats. Life Sciences 199557 2081-2090.

34 Lundquist I. Cholinergic muscarinic effects on insulin release in mice. Pharmacology $198225338-347$.

35 Bellavere F, Cacciatori V, Moghetti P, Gemma ML, Dellera A, Tosi F et al. Acute effect of insulin on autonomic regulation of the cardiovascular system: a study by heart rate spectral analysis. Diabetic Medicine 199613 709-714.

36 Gros R, Borkowski KR \& Feldman RD. Human insulin-mediated enhancement of vascular beta-adrenergic responsiveness. Hypertension 199423 551-555.

37 Lembo G, Iaccarino G, Vecchione C, Rendina V, Parrella L \& Trimarco B. Insulin modulation of beta-adrenergic vasodilator pathway in human forearm. Circulation 199693 1403-1410.

38 Veldhuis JD, Faria A, Vance ML, Evans WS, Thorner MO \& Johnson ML. Contemporary tools for the analysis of episodic growth hormone secretion and clearance in man. Acta Paediatrica Scandinavica $198834763-82$.

39 Merriam GR \& Wachter KW. Algorithms for the study of episodic hormone secretion. American Journal of Physiology 1982243 E310-E318.

Received 26 February 1999

Accepted 13 May 1999 